Use of Hypnotics in Older People with Mental Illness: A Systematic Study of Tolerability and Use in Different Diagnostic Groups

Stephen Curran ${ }^{1}$, Debbie Turner ${ }^{2}$, Shabir Musa $^{2}$, Andrew Byrne $^{2}$, John Wattis ${ }^{3}$

${ }^{1}$ University of Huddersfield and South West Yorkshire Mental Health NHS Trust

${ }^{2}$ South West Yorkshire Mental Health NHS Trust

${ }^{3}$ University of Huddersfield 


\title{
Use of Hypnotics in Older People with Mental Illness: A Systematic Study of Use in Different Diagnostic Groups
}

\author{
Stephen Curran, Debbie Turner, Shabir Musa, Andrew Byrne, \\ John Wattis
}

\begin{abstract}
Aims The objective of the study was to provide observational clinical data on psychotropic drugs and especially hypnotics used in older people with mental illness.
\end{abstract}

Method This was an observational, single-centre, one-week prevalence study of psychiatric symptoms, disorders and psychotropic/hypnotic drug use in older people with mental illness cared for by the South West Yorkshire Mental Health NHS Trust (Wakefield Locality), UK.

Results A total of $593 / 660$ older patients with mental illness (mean \pm SD age, $76 \pm 8.1$ years) were assessed). $44.5 \%$ had dementia (excluding vascular dementia) and $33.7 \%$ had a mood disorder. Of the total, $20.4 \%$ did not receive CNS active medication and $46.2 \%$ of patients were prescribed an antidepressant. In total $130(21.9 \%)$ of patients were prescribed an hypnotic and these were more likely to be prescribed to patients with depression (35\%) compared with other diagnoses (vascular dementia $=17.2 \%$, dementia $=12.1 \%$, schizophrenia and related disorders $=21.5 \%$ and anxiety disorders $=27.3 \%) \quad\left(X^{2}=36.3, \quad p<0.001\right)$. Zopiclone was the most commonly prescribed hypnotic accounting for $55 \%$ of all hypnotic prescriptions and was most commonly prescribed to patients with depression $\left(X^{2}=30.4, p<0.001\right)$.

Conclusions: Hypnotics are commonly used in older people with mental illness including depression, dementia, schizophrenia and anxiety disorders. They are significantly more likely to be prescribed to patients with depression compared with other diagnostic groups and zopiclone was the most commonly prescribed hypnotic accounting for $55 \%$ of hypnotic prescriptions. Hypnotics were generally well tolerated and patients were broadly satisfied with their medication.

Key words: psychotropic's, hypnotics, older people, mental illness

\section{Introduction}

Insomnia is a common complaint affecting approximately one-third of the UK population in any one year. It is more common in women, in the elderly and in those with psychiatric disorders (Shapiro, 1993). More than 10 million prescriptions for hypnotics continue to be dispensed each year in England alone, mostly for benzodiazepines and drugs with similar actions such as zaleplon, zolpidem and zopiclone (so-called "Z-drugs") (Department of Health, 2005). Approximately $80 \%$ of these prescriptions are for those aged 65 years or older (Curran et al., 2003). Despite recommendations for short term use, a significant proportion of people remain on these drugs for months or even years (Taylor, et al., 1998). 
Primary insomnia is defined as a difficulty initiating or maintaining sleep or experiencing non-restorative sleep that results in clinically significant distress or impairment in functioning (America Psychiatric Association, 1994) (Table 1). For a diagnosis of primary insomnia, the sleep problem cannot be attributed to a medical, psychiatric, or environmental cause (such as drug abuse or medications) whereas secondary insomnia denotes insomnia due to causes such as these.

\section{Table 1: DSM-IV Diagnostic Criteria for Primary Insomnia}

A. Difficulty with initiating or maintaining sleep, or non restorative sleep, for at least 1 month.

B. The sleep disturbance (or associated daytime fatigue) causes clinically significant distress or impairment in social, occupational, or other important areas of functioning.

C. The sleep disturbance does not occur exclusively during the course of narcolepsy, breathing-related sleep disorder, circadian rhythm sleep disorder, or a parasomnia.

D. The disturbance does not occur exclusively during the course of another mental disorder (for example, major depressive disorder).

E. The disturbance is not due to the direct physiological effects of a substance (e.g., a drug of abuse, a medication) or a general medical condition.

The cause of insomnia is important as this will influence management and may help avoid having to use drug treatments (Maczaj, 1993). Causes of insomnia include physiological (e.g. shift-workers and excessive exercise), psychological (e.g. distressing life events), psychiatric (e.g. depression, anxiety and dementia), physical (e.g. poorly managed pain), pharmacological (e.g. stimulant drugs) and idiopathic (primary insomnia) (Wheatley, 1986). The risk of primary insomnia increases with age and may be related to changes in circadian rhythms also associated with increasing age (Bliwise, 1993).

The management of insomnia includes having a clear understanding of the aetiology and managing this appropriately. For primary insomnia simple measures should be tried first and attention to sleep hygiene (e.g. room temperature, ambient lighting and sound levels, diet etc) can be very effective. Pharmacological treatments should only be tried when these simple measures have been tried. The ideal hypnotic would induce sleep rapidly, keep patients asleep without disrupting sleep architecture, have no adverse effects, produce no next-morning sedation, lack dependence and rebound insomnia, and be safe in the medically unwell and in overdose. No drug satisfies all these criteria so these drugs need to be used with caution, especially in older people. Guidance from national advisory bodies has recommended that hypnotics should only be used in the short-term for the treatment of insomnia (Royal College of Psychiatrists, 1997, National Institute for Health and Clinical Excellence (NICE), 2004, Joint Formulary Committee, 2004). In April 2004, NICE guidance was also published on the use of zaleplon, zolpidem and zopiclone for the treatment of insomnia. 


\section{Table 2: NICE Guidance on zaleplon, zolpidem and zopiclone for the management of insomnia}

- NICE recommends that doctors should consider using nonmedicine treatments, and then, if they think that a hypnotic medicine is the appropriate way to treat severe insomnia that is interfering with normal daily life, they should prescribe one for only short periods of time and strictly according to the licence for the drug.

- Because there is no firm evidence of differences in the effects of zaleplon, zolpidem, zopiclone and the shorter-acting benzodiazepines, NICE recommends that doctors should prescribe the cheapest drug, taking into account the daily dose required and the cost for each dose.

- Treatment should only be changed from one of these hypnotics to another if side effects occur that are directly related to the medicine.

- If treatment with one of these hypnotic medicines does not work, the doctor should not prescribe one of the others.

Benzodiazepines replaced barbiturates as the main treatment for primary insomnia over the past 30 years or so. Although these drugs were initially welcomed, problems arose with their indiscriminate use. Hypnotics may be classified in a variety of ways and no one approach is universally accepted. However, the classification into benzodiazepine and non-benzodiazepine drugs is clinically useful (Hindmarch and Currie, 1995). Classification based on half-lives is also clinically meaningful e.g. short-acting (oxazepam) and longer acting (nitrazepam). The gamma-aminobutyric acid (GABA) receptor is the site of action of most hypnotic drugs (Doble, 1999) but classification based on sites/modes of action are not very helpful from a clinical perspective (Vermeeren, 2004).

A detailed review of hypnotics is beyond the scope of this paper. However, there is a good evidence base for hypnotic drug use in younger people but less so in relation to older people despite the fact that most prescriptions for hypnotics are for older people. A meta-analysis of 24 randomised controlled trials (involving 2417 participants) compared potential benefits and risks of short-term treatment with sedative hypnotics in people aged 60 years or over with insomnia (Glass 2005). The authors concluded that improvements in sleep were statistically significant. However, the increased risk of adverse events was also statistically and clinically significant including falls and cognitive impairment. The authors queried whether the clinical benefits could be justified in view of the prevalence and severity of the adverse events. There have also been a number of studies which have focused specifically on older people (Barbera 2005; Duran 2005; Melton, 2005; Moen et al 2006; Halas 2006).

Despite the availability of both established and newer hypnotics for the treatment of insomnia, further research is necessary to inform the evidence base for best clinical use in older people. The aim of the present study was 
to provide a better understanding of hypnotic drug use in older people with mental illness and particularly hypnotic drug use in different diagnostic groups.

\section{Method}

\section{Study design}

This was an observational, single-centre, one week prevalence study of psychiatric symptoms, disorders and psychotropic drug use, especially hypnotic drug use carried out in the Wakefield District (UK), South West Yorkshire Mental Health NHS Trust, UK over 12 months in 2003/2004. The population in the District consisted of approximately 55,000 people aged 65 years or older. The service provided for this population consisted of two acute wards, one day-hospital, outpatient clinics for three consultant teams, three Community Units for the Elderly, and two Community Mental Health Teams. The study was approved by the Wakefield Research Ethics Committee.

\section{Patient selection}

All consenting patients under the care of psychiatric services for older people in the Wakefield District were included in the study. Patients identified from Trust records were contacted by a Research Nurse to ask if they would like to take part in the study. All patients and caregivers received an information sheet before taking part in the study and gave written consent.

\section{Assessments}

The Research Nurse undertook a detailed clinical assessment. The clinical assessment lasted approximately one hour and included demographic details, history of current and previous mental illness, a history of past and current physical illness, diagnosis, treatment response (classified as first episode, stable-dissatisfied, stable-satisfied, treatment resistant, and uncontrolled) and medication use and tolerability. In addition drug sideeffects were also assessed using the Psychosis Evaluation Tool for Common use by Caregivers (PECC) (Lindstrom et al., 1997). The PECC was specifically designed to be used by health care workers including nurses and evaluates a range of drug side-effects. The reliability and validity of PECC has been described in both younger and older people (de Hert et al., 1999).

The clinical assessment also included an interview with the caregiver, discussions with medical and nursing staff and a review of medical notes including GP records. This specifically included a review of patients' current physical health and laboratory and other investigations.

Prior to undertaking the study the Research Nurse attended a three-day training course to standardise the clinical assessment procedures.

Patients were assessed in a variety of settings including the two acute wards, Out Patient (OP) clinics, the three Community Units for the Elderly and in their own homes. The assessment took approximately one hour to 
complete and after the assessment a copy was made available to the appropriate clinical team. Diagnosis was based on DSM-IV criteria (APA, 1994). Some patients attended several parts of the service e.g. day hospital and OP clinic but they were only included once.

Side-effects were based on the previous seven days and a standardised protocol was used for defining and scoring individual side-effects. Sideeffects were measured on a four-point scale (1=absent; $4=$ =severe, obvious influence on functioning, intervention necessary).

\section{Statistical analysis}

Statistical analyses were carried out using SAS/STAT software (version 8.12). Comparisons of continuous variable used ANOVA, and pair-wise comparisons (Chi squared test - $\chi^{2}$, Cochran-Mantel-Haenzel test) for categorical variables were performed with adjustment for multiple comparisons employing the Tukey-Kramer's method.

\section{Results}

\section{Patient characteristics}

Of a total of 660 older patients, $593(89.8 \%)$ patients took part in the study. 293 patients (approximately 50\%) had a diagnosis of dementia with $4.9 \%$ of the total population having vascular dementia $(\mathrm{VaD})$. Of the remaining patients $200(33.7 \%)$ had an affective disorder and $65(11 \%)$ schizophrenia or a related disorder. In addition, the majority of patients had had their mental illness for a relatively short mean period -0.4 years for patients with $\mathrm{VaD}$ and 1.7 years for patients with schizophrenia and related disorders. Sixty-nine percent $(n=409)$ of patients were female and there were more females $(\geq 67 \%)$ in each diagnostic category $\left(\chi^{2}, p=0.001\right)$, with the exception of $\mathrm{VaD}$ dementia (males $\mathrm{n}=19,65.5 \%$; females $n=10,34.5 \%$ ).

\section{Psychoactive drugs}

Of the 593 patients, $121(20.4 \%)$ did not receive a psychoactive drug. A total of $304(51.3 \%)$ patients were taking an antipsychotic, $274(46.2 \%)$ an antidepressant, $125(21.1 \%)$ an antidementia drug, $29(4.7 \%)$ an anticonvulsant, 29 (4.9\%) anticholinergic drug, 42 (7.1\%) an anxiolytic and $130(21.9 \%)$ a hypnotic.

\section{Intake of hypnotics}

In total $130(21.9 \%)$ of patients were prescribed an hypnotic and these were more likely to be prescribed to patients with depression $(35 \%)$ compared with other diagnoses $(\mathrm{VaD}=17.2 \%$, dementia $=12.1 \%$, schizophrenia and related disorders $=21.5 \%$ and anxiety disorders $=27.3 \%$ ) $\left(X^{2}=36.3, p<0.001\right)$. Zopiclone was the most commonly prescribed hypnotic accounting for $55 \%$ of all hypnotic prescriptions. Zopiclone was most commonly prescribed to patients with depression (22\%) compared with vascular dementia (10.3\%), dementia $(5.7 \%)$, schizophrenia and related disorders $(7.7 \%)$ and anxiety disorders $(15.2 \%)$ and these differences were significantly different $\left(X^{2}=30.4, p<0.001\right)$. The five most commonly prescribed hypnotics are listed in Table 3. In addition, one patient was 
using phenobarbitone (long-term) and one patient low dose amitriptyline as hypnotics.

Table 3: The five most commonly prescribed hypnotics in 593 older people with mental illness (mean and median doses in $\mathrm{mg}$ )

\begin{tabular}{|l|l|l|l|}
\hline Drug & $\mathbf{N}$ & Mean dose in $\mathbf{m g}$ & Median dose in mg \\
\hline Zopiclone & 70 & 11.9 & 7.5 \\
\hline Temazepam & 29 & 13.3 & 10 \\
\hline Nitrazepam & 15 & 7.2 & 5 \\
\hline Diazepam & 9 & 5 & 5 \\
\hline Zolpidem & 5 & 6 & 5 \\
\hline
\end{tabular}

\section{Evaluation of side-effects}

There was a statistically significant difference between the different diagnostic groups for the mean scores of anticholinergic side-effects $(F=2.9, p=0.02)$ and EPS $(F=15.6, p<0.001)$. Patients with schizophrenia and related disorders had significantly higher levels of anticholinergic and EPS side-effects. Hormonal side-effects were not significantly different between the diagnostic groups $(F=0.3, p=0.87)$.

Hypnotic's drugs were generally well tolerated with very few reported sideeffects and no serious adverse events were recorded. No patient was prescribed medication outside British National Formulary (BNF) recommended doses. Drowsiness the following morning was the most common side-effect. This was usually mild but was significantly more likely to occur in patients with depression and the degree of following-morning drowsiness was significantly different from other diagnostic groups (ANOVA, $\mathrm{F}=7.8, \mathrm{p}<0.001$ ). In addition, treatment response and patient satisfaction to hypnotic use were rated as "satisfied" for the majority of patients $(n=121,93.1 \%)$ with 6 patients $(4.6 \%)$ rated as "dissatisfied" and only 3 patients $(2.3 \%)$ rated as "very dissatisfied".

\section{Discussion}

The use of hypnotics in older people is a relatively neglected area of research particularly in clinical populations. The recent NICE publication is welcome but it makes no specific mention of hypnotic use in older people and particularly those with mental illness.

The principal objective of this study was to obtain a better understanding of the use and tolerability of hypnotic drug use in older people with mental illness in the wider context of psychotropic drug use and in different psychiatric diagnostic groups.

This study confirms that hypnotics are commonly prescribed to older people with mental illness with $21.9 \%$ of all patients prescribed one of these drugs. 
Patients with depression were most likely to be prescribed a hypnotic (35\%) but patients in other diagnostic groups were also commonly prescribed these drugs including vascular dementia (17.2\%), Alzheimer's disease $(12.1 \%)$, schizophrenia and related disorders $(21.5 \%)$ and anxiety disorders $(27.3 \%)$. In addition, hypnotics were generally well tolerated but patients with depression were more likely to experience following-morning drowsiness compared with other diagnostic groups. This might be because patients with depression often experience early morning tiredness irrespective of hypnotic drug use. The range of hypnotics used was relatively narrow and only five were commonly used including zopiclone, temazepam, nitrazepam, diazepam and zolpidem. Use of diazepam as a hypnotic was concerning because of its very long half-life in older people but evidence for major following-morning drowsiness was not found. In addition, the mean dose of diazepam was only $5 \mathrm{mg} /$ day which is relatively low. Only one patient was prescribed low dose amitriptyline as a hypnotic and one patient continued to take phenobarbitone as a hypnotic. Phenobarbitone had been prescribed for several decades and had been continued because of difficulties withdrawing the drug. The use of low dose TCAs has been debated in the management of insomnia but the evidence base is very limited and the use of tricyclic antidepressants is not generally recommended for the management of insomnia. Reassuringly, no patient was prescribed more than one hypnotic and all patients were prescribed within BNF recommended limits. In addition, over $90 \%$ of patients reported feeling "satisfied" with their hypnotic treatment.

In this study hypnotics were prescribed within BNF limits, side-effects were mild and the majority of patients were satisfied with their treatment. Drugs such as diazepam should probably be avoided in the management of insomnia because of the potential for significant next morning drowsiness. A greater emphasis should be placed on identifying treatable causes such as depression combined with patient education and sleep hygiene advice. If drugs are used the $z$ drugs are preferable including zopiclone, zolpidem and zaleplon but they should be prescribed only for relatively short periods. Unfortunately most of the research in this area has focused on younger people. More research specifically in older people with mental illness is needed if the management and particularly the pharmacological management of insomnia in this neglected group are to gain greater prominence and become more evidence based.

\section{Acknowledgements}

This was an Investigator Initiated Project funded by an unconditional educational grant from Janssen-Cilag (UK). 


\section{References}

American Psychiatric Association (1994) Diagnostic and Statistical Manual of Mental Disorders, Third Edition (Revised), Washington, D.C.

Barbera, J. \& Shapiro, C, 2005 Benefit-risk assessment of zaleplon in the treatment of insomnia. Drug Safety, 28(4):301-18.

Bliwise, D.L. 1993 Sleep in normal aging and dementia. Sleep, 16:40-81.

Curran, H.V., Collins, R., Fletcher, S., Kee, S.C.Y \& Woods, B., Iliffe, S. 2003. Older adults and withdrawal from benzodiazepine hypnotics in general practice: effects on cognitive function, sleep, mood, and quality of life. Psychological Medicine, 33: 1223-37.

De Hert, M., Bussels, J., Lindstrom, E., Abrahams, F., Fransens, C. \& Peuskens, J. 1999. PECC Psychosis Evaluation Tool for Common use by Caregivers. Drukkerji EPO: Berchem.

Department of Health 2005. Prescription costs analysis (PCA). 2005 [online]. http://www.ic.nhs.uk/pubs/prescostanalysis2005/final/file

Doble A. 1999. New insights into the mechanism of action of hypnotics. Journal of Psychopharmacology, 13(4 Suppl 1): S11-20.

Duran, J. C., Greenspan, A., Diago, J.I, Gallego, R. \& Martinez, G. 2005. Evaluation of risperidone in the treatment of behavioral and psychological symptoms and sleep disturbances associated with dementia. International Psychogeriatrics, 17(4):591-604.

Glass, J. Lanctôt, K.L., Herrmann, N., Sproule, B.A. \& Busto, U.E. 2005. Sedative hypnotics in older people with insomnia: meta-analysis of risks and benefits. British Medical Journal, 331:1169.

Halas, C.J. 2006 Eszopiclone. American Journal of Health-System Pharmacy, 63(1):41-8.

Hindmarch, I. \& Currie, D.J. 1995. Pharmacokinetics, pharmacodynamics and drug development. In Seminars in Clinical Psychopharmacology, King DL (ed.).Gaskell: London, 27-58.

Joint Formulary Committee. British National Formulary. Edition 51. London: Royal Pharmaceutical Society of Great Britain and British Medical Association, March 2006.

Lindstrom, E., Ekselius, I., Jedenius, E., Almqvist, A. \& Wieselgren, I.M. 1997. A checklist for assessment of treatment in schizophrenia syndromes; interscale validity and interrater reliability. Primary Care Psychiatry 3: 183187.

Maczaj, M. 1993. Pharmacological treatment of insomnia. Drugs, 45(1):4455. 
Melton, S.T. \& Wood, J.M. 2005. Eszopiclone for insomnia. Annals of Pharmacotherapy, 39(10):1659-66.

Moen, M.D. \& Plosker, G.L. 2006. Zolpidem extended-release. CNS Drugs, 20(5):419-426.

National Institute for Health and Clinical Excellence (NICE) 2004. Insomnia - newer hypnotic drugs: zaleplon, zolpidem and zopiclone for the management of insomnia. Technology Appraisal Guidance 77, 2004. (Reference: TA077) www.nice.org.uk

Royal College of Psychiatrists 1997. Benzodiazepines: Risks, Benefits or Dependence: a re-evaluation. Council Report CR59. London: Royal College of Psychiatrists.

Shapiro, C.M. (Ed.). 1983. ABC of Sleep Disorders. London: BMJ Publishing Group.

Taylor, S. et al. 1998. Extent and appropriateness of benzodiazepines use. Results from an elderly urban community. British Journal of Psychiatry 1998; 173: 433-8.

Vermeeren, A. 2004. Residual effects of hypnotics. Epidemiology and clinical implications. CNS Drugs, 18: 297-328.

Wheatley, D. 1986. Insomnia in general practice: the role of temazepam and a comparison with zopiclone. Acta Psychiatrica Scandinavica Suppl. $332: 142-8$ 